\title{
Bone biology \& pathology moves on: from bone resorption to formation, the rise of new therapeutic opportunities and experimental tools
}

Dear Readers,

Bone comprises a complex mineralized connective tissue characterized by the constant remodeling, which involves cycles of bone resorption coupled with the subsequent bone formation. Also, bone tissue retains a considerable potential for healing, which also involves the cooperative action of bone forming and resorptive cells to restore the architecture and function of damaged tissue. However, pathological conditions such as bone cancer and infectious inflammatory conditions (such as periodontal and periapical lesions) may disturb the resorption/formation coupling process, resulting in irreversible bone destruction.

Despite the recent huge advances on the understanding of bone biology and pathology, specially driven by the discovery of the RANK/ RANKL/OPG system, composed of molecules shared by bone and immunological systems (that ultimately resulted in the raise of the 'osteoimmunology' terminology), a series of open questions remain unanswered in both bone resorption and formation ends $^{5}$. In this issue of the Journal of Applied Oral Science (JAOS), we are pleased to present examples of the evolving bone science, describing new therapeutic opportunities and experimental tools.

Starting with the new experimental tools, Araujo, et al. ${ }^{1}$ (2015) use an experimental tooth movement model to insightfully demonstrate that fractal analysis can be useful in the evaluation of osteoclastic activity. Using classical histological tools to identify osteoclasts as a control, the authors demonstrate a positive correlation between osteoclasts counts and fractal dimension obtained with the new methodology described. Also, the inhibition of osteoclasts formation and activity by means of alendronate administration also impacted the fractal dimension measurement, proving the validity of the proposed method in a cause-andeffect manner.

Another important new experimental tool described in this issue comprises a very important advance in the study of mandibular bone metastasis. Previously in the JAOS ${ }^{7}$, a case of mandibular bone metastasis derived from a tumoral prostate adenocarcinoma was described. However, animal models of mandibular bone metastasis, which could comprise an important tool to understand the molecular mechanism of mandible bone metastasis and to develop new therapeutic agents, are scarce. In this context, in this issue, Hwang, et al. ${ }^{6}$ (2015) describe a pre-clinical mouse model for metastatic breast cancer to the mandible. Using bioluminescence, micro-computed tomography and histopathological analysis, the authors demonstrate that extensive mandibular bone destruction occurred in nude mice grafted with luciferasetransfected metastatic breast cancer cells (MDAMB-231LuC+), and validate the new model as a useful tool to advance the studies on the osteolytic cancers field.

Moving the focus to new therapeutic opportunities, Saglam, et al. ${ }^{8}$ (2015) use an established experimental periodontitis model to demonstrate that Sumac (Rhus coriaria L.), a widely used spice, can control the progression of periodontal lesions. Using histometric, immunohistochemical and enzymatic methods, the authors demonstrate that the sumac extract, administered orogastrically, inhibits periodontitis development via the regulation of oxidative status and RANKL/OPG system, opening the opportunity to possible clinical application to manage periodontitis. Also, in line with previous reports previously published in the JAOS ${ }^{2,3}$, this study reinforces the central role of RANKL/OPG system in the pathogenesis of bacterially induced inflammatory osteolytic lesions, as well supports the validity of modulating host responses strategies to arrest periodontitis ${ }^{9}$.

Finally, still in the therapeutic end, Ferraz, et al. ${ }^{4}$ (2015) demonstrate that plasma-nitrided titanium implant surfaces result in successful osteointegration in rabbits. By means of histological and histomorphometric analyses, the authors demonstrate that bone formation was observed in contact with all plasma-nitrided titanium implants; being such response similar to those observed in the positive controls. Based on the data obtained, the authors conclude that since plasma nitriding treatments improve the physico-chemical properties of Ti without affecting its biocompatibility, they could be used along with other modifications, such as functionalization with growth factors, to improve bone formation in implant therapy.

Therefore, the four studies mentioned are stimulating examples of the evolving bone science, which using new experimental tools can improve the understanding of the mechanisms underlying bone resorption and formation in physiological, pathological and regenerative scenarios, and apply this knowledge to the subsequent development 
of new therapeutic opportunities to control tissue damage or promote its regeneration.

\section{References}

1- Araujo AS, Fernandes ABN, Maciel JVB, Santos JN Netto, Bolognese AM. New methodology for evaluating osteoclastic activity induced by orthodontic load. J Appl Oral Sci. 2015;23(1):19-25. 2- Araujo-Pires AC, Francisconi CF, Biquetti CC, Cavalla F, Aranha $A M$, Letra $A$, et al. Simultaneous analysis of $T$ helper subsets (Th1, Th2, Th9, Th17, Th22, Tfh, Tr1, and Tregs) markers expression in periapical lesions reveals multiple cytokine clusters accountable for lesions activity and inactivity status. J Appl Oral Sci. 2014;22(4):336-46.

3- Benatti BB, Campos-Júnior JC, Silva-Filho VJ, Alves PM, Rodrigues IR, Uber-Bucek $E$, et al. Effects of a Mikania laevigata extract on bone resorption and RANKL expression during experimental periodontitis in rats. J Appl Oral Sci. 2012;20(3):340-6.

4- Ferraz EP, Sverzut AT, Freitas GP, Sá JC, Alves C Jr, Beloti MM, et al. Bone tissue response to plasma-nitrided titanium implant surfaces. J Appl Oral Sci. 2015;23(1):9-13.

5- Garlet GP. Destructive and protective roles of cytokines in periodontitis: a re-appraisal from host defense and tissue destruction viewpoints. J Dent Res. 2010;80(12):1349-63.
6- Hwang YS, Han SS, Kim KR, Lee YJ, Lee SK, Park, KK, et al. Validating of the pre-clinical mouse model for metastatic breast cancer to the mandible. J Appl Oral Sci. 2015(1):3-8.

7- Menezes JD, Capellari PF, Capelari MM, Gonçalves PZ, Toledo GL, Toledo GL Filho, et al. Mandibular metastasis of adenocarcinoma from prostate cancer: case report according to epidemiology and current therapeutical trends of advanced prostate cancer. J Appl Oral Sci. 2013;21(5):490-5.

8- Sağlam M, Kőseoğlu S, Hatipoğlu M, Esen HH, Kőksal E. Effect of sumac extract on serum oxidative status, RANKL/OPG system and alveolar bone loss in experimental periodontitis in rats. J Appl Oral Sci. 2015;23(1):33-41.

9- Souza JA, Rossa C Jr, Garlet GP, Nogueira AV, Cirelli JA. Modulation of host cell signaling pathways as a therapeutic approach in periodontal disease. J Appl Oral Sci. 2012;20(2):12838.

\section{Gustavo Pompermaier Garlet Editor-in-Chief Journal of Applied Oral Science}

